\title{
THE ROLE OF PARENTAL REARING STYLES IN THE PERCEPTION OF COLLEGE ADOLESCENTS' ANXIETY SYMPTOMS
}

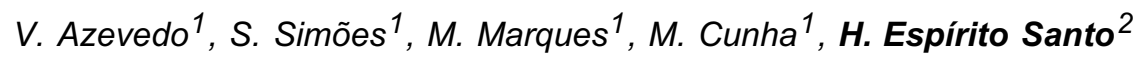 \\ ${ }^{1}$ Instituto Superior Miguel Torga, ${ }^{2}$ CEPESE/Centro de Investigação e Desenvolvimento, Miguel Torga Higher Institute, \\ Coimbra, Portugal
}

The literature highlights the importance of the impact of parental behavior on the development of anxiety in children and adolescents. The aim of this study was to analyze the role that the adolescents' perception about parental rearing styles have on the manifestation of anxiety symptoms.

The sample involved 136 college adolescents, 48 boys and 88 girls aged between 12 and 15 years (M=13,2 years). The research protocol included Sociodemographic Questionnaire, State-Trait Anxiety Inventory for Children (STAIC) and the EMBU-A.

Results suggest that older adolescents (14-15 years) showed greater anxiety symptoms, significant at the level of state anxiety. The young people with more academic failure externalize more trait anxiety and perceive higher levels of mother's overprotection and rejection of father and mother. Adolescents who have a father with lowest educational level perceive greater maternal rejection, and are the children of mothers with less qualification who feel greater mothers' overprotection and father's rejection. There was a association between paternal rejection and higher levels of anxiety symptoms. The predictive model confirms that parental rejection, together with the age of the adolescent, are significant predictors of anxiety symptoms. Specifically, parental rejection is evidenced as the best predictor of anxiety symptoms, being primarily responsible for the manifestation of anxiety in adolescents.

These results suggest that father's rejection generates high levels of anxiety symptoms. Thus, this study shows that rejection is the paternal parental rearing style that has more influence on the manifestation of anxiety in adolescents, triggering high levels of anxiety. 